\title{
MODELLING THE EVOLUTION OF THE EAST ANTARCTIC ICE SHEET: PROBLEMS AND PERSPECTIVES (Abstract)
}

\author{
by
}

\author{
S.S. Grigoryan, I.Yu. Ignat'yeva, M.S. Krass and P.A. Shumskiy \\ (Moskovskiy Gosudarstvennyy Universitet, Institut Mekhaniki, \\ Michurinskiy Prospekt 1, 117234 Moscow, U.S.S.R.)
}

\begin{abstract}
Modelling the thermodynamics of a large ice sheet is in essence a problem of the deformation of a nonisothermal and relatively thin viscous layer. Reasons for forecasting the behaviour of a large ice sheet are: (a) to specify the relationship between the existence and development of the ice sheet and regional climatic and tectonic conditions; (b) to study the influence of variations in surface temperature, mass balance, and geothermal flux (as functions of coordinates and time) on the evolution of the thickness of the ice sheet. Hence the principal requirements for modelling are:

1. The model must be evolutionary; in particular, various stationary stages should be derived from it (rather than postulated).

2. The model must be non-isothermal because temperature variations amount to some tens of degrees.

3. Two-dimensionality of the model is essential if we are to use it for making realistic forecasts.

4. Conditions at the boundary between land ice and the sea, which are the basic factors controlling the location of the margin of the ice sheet, should be taken into account.

5. Finally, the model must be acceptable for digital analysis by computer.

Contemporary mathematical models of the dynamics of the Antarctic and Greenland ice sheets are too simplified
\end{abstract}

and do not fulfil these requirements. Our mathematical model of the evolution of the Antarctic ice sheet is based on temperature parameterization. The parameterization correctly describes the distribution of temperature and its dependence on surface temperature, advection of ice, geothermal flux, and heat losses. The problem reduces to Cauchy's problem for a non-linear parabolic partial differential equation with three-step dependence of ice temperature on depth. A linear viscous flow law is assumed. Tests treat the whole problem of the thermo-hydromechanics of the ice sheet. The ice sheet is assumed to be bounded by a grounding line or ice wall where the ice is in hydrostatic equilibrium with the sea. In this case, the problem reduces to a non-linear parabolic partial differential equation with a complicated boundary condition at the moving edge. A digital representation of the model is presented first. Tests demonstrated the possibility of using an explicit three-layered scheme. Calculations for the East Antarctic ice sheet indicated that the ice margin should retreat slightly under contemporary climatic conditions (which corresponds to the field evidence). In future the boundary should tend towards a stationary state. A forecast of the behaviour of the ice sheet is derived. The East Antarctic ice sheet is found to be stable during various changes in climatic factors. The calculated hydromechanical characteristics of the ice sheet agree with the observed field data. 